\title{
BINOCULARITY IN INFANCY
}

\author{
OLIVER BRADDICK \\ London
}

\begin{abstract}
SUMMARY
A variety of behavioural and electrophysiological studies agree that the onset of functional binocular interaction in human visual cortex normally occurs between 10 and 16 weeks of age. Measures of sensitivity to binocular correlation and to disparity agree closely, and behavioural and visual evoked potential measures on the same infant show onset of binocularity within about a 2 week range. Beyond the initial onset, the maximum disparity to which infants are sensitive increases steadily and stereoacuity is found to increase very rapidly. The initial development of binocularity does not appear to be a consequence of improving alignment of the eyes and occurs even in the presence of strabismus. However, the connections subserving binocularity are plastic in early childhood; they can be disrupted by unilateral strabismus, although in some strabismic children who use both eyes for fixation, they can adapt to serve stereo function at the angle of deviation and re-adapt, albeit temporarily, to the surgical alignment of the eyes. These findings allow us to pose some as yet unanswered questions about the development of binocularity, including: How is the infant's visual system organised before the establishment of binocularity? How does the pre-binocular infant maintain vergence? And what neural changes underlie the increase in performance for small and large disparities following the initial onset of binocular function?
\end{abstract}

\section{THE ONSET OF BINOCULARITY}

It is now possible to present a fairly clear and well agreed outline of how binocularity develops in infancy. A number of different behavioural and electrophysiological methods agree that the onset of binocular interaction in the human visual cortex is between 10 and 16 weeks of age in most infants (see reviews by Braddick and Atkinson ${ }^{1}$ and Birch $^{2}$ ). However, there is considerable real individual variation within the range of at least $8-20$ weeks. ${ }^{3,4}$

Correspondence to: Prof. O. J. Braddick, Visual Development Unit, Department of Psychology, University College London, Gower Street, London WC1E 6BT, UK.
The first method used in our laboratory was to record visual evoked potentials (VEPs) when the infant watched a 'dynamic random-dot correlogram ${ }^{5,6}$ This display consists of a random pattern of red and green dots which are changed completely on each video frame. Two phases alternate: in one the red and green dot patterns are identical (correlated), and in the other the green pattern is the negative of the red (anticorrelated). The infant wears red-green goggles. Each eye individually sees a pattern in which the alternations are undetectable, since the pattern change at alternation is no different from that which occurs on every other frame. However, cortical neurons which integrate signals from the two eyes will respond to the difference between the correlated display that can be fused
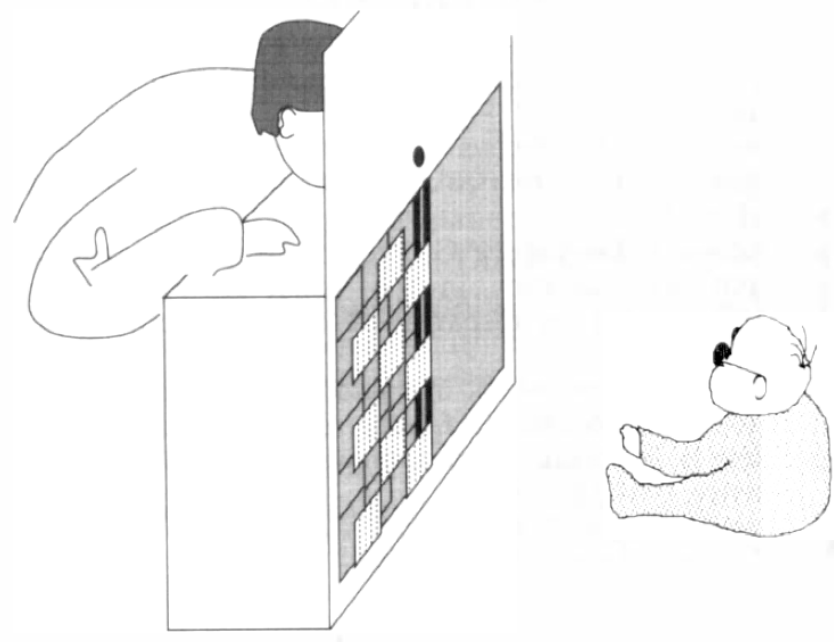

Fig. 1. Arrangement used by Smith et al. ${ }^{7,8,29}$ for testing infant binocularity by forced-choice preferential looking (FPL). The infant wears lightweight goggles with red and green filters in order to separate dynamic red/green dot patterns displayed on a large video monitor. In the stereogram display, the checkerboard pattern shown represents regions which have a crossed disparity (typically 42 min arc) relative to the background; they are not visibly distinctive if seen by either eye alone. These checks alternate between zero and crossed disparity at 2 alternations per second. The correlogram display is similar except that the checks alternate between correlated and anticorrelated dots. 


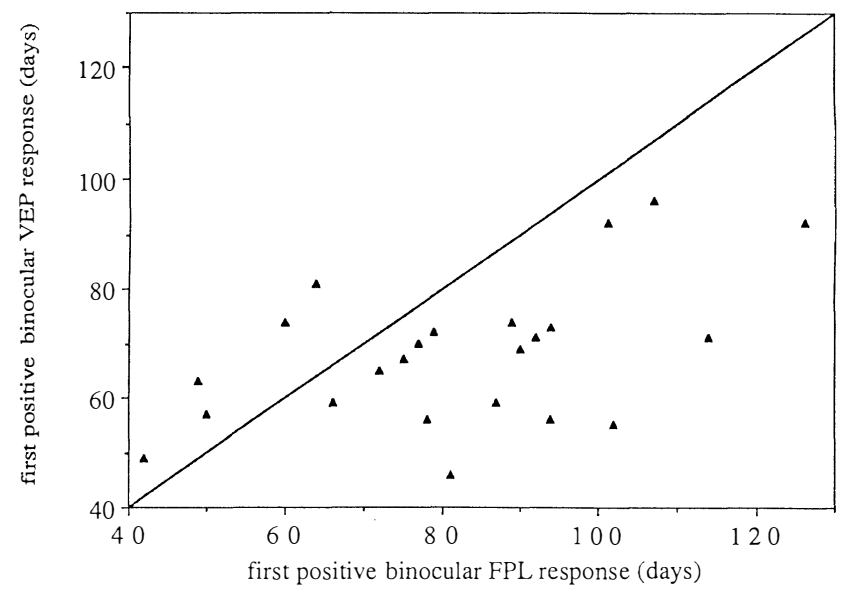

Fig. 2. Comparison in individual infants $(\mathrm{n}=24)$ of the age of onset of FPL and VEP responses to random-dot stimuli. Each infant was tested with FPL and VEP methods on alternate weeks; correlogram and stereogram stimuli were both tested in each session. Whether VEP or FPL was used in the first week of testing was counterbalanced across infants. The points mark the age at which the first statistically significant response with either stimulus was obtained (VEP, $\mathrm{p}<0.05$ on the circular variance test; ${ }^{41} \mathrm{FPL}$, $\mathrm{p}<0.05$ on binomial test); the diagonal line marks equal ages on the two measures. The mean age for first FPL response was 82 days; for first VEP response, 68 days (difference significant $\mathrm{p}<0.002$, two-tailed $\mathrm{t}$-test). (From Smith. $\left.{ }^{7}\right)$

perfectly, and the uncorrelated display which cannot. This response can be detected as a periodic VEP at the alternation frequency, whose emergence can be tracked longitudinally in individual infants. ${ }^{3,4}$

The findings are not closely dependent on how the infant's response to correlation-anticorrelation is measured. Similar displays can be used in a forcedchoice preferential looking (FPL) test: one half of the display remains uniformly anticorrelated, while the other half contains large checks which alternate between correlation and anticorrelation (Fig. 1). In a longitudinal study in our laboratory ${ }^{7,8}$ the same infants were tested on alternate weeks with the VEP and FPL methods. There was a strong correlation between the ages at which a statistically reliable response of each kind was detected (Fig. 2), with the average age of onset being about 2 weeks later on the FPL method compared with the VEP method.

\section{STEREOPSIS AND CORRELATION}

Strictly, positive performance on the correlogram test is not a demonstration of stereopsis. Stereopsis requires the ability not only to distinguish correlation from non-correlation, but to distinguish correlation at different disparities. However, Smith's longitudinal study also tested infants with two different displays: the correlogram stimulus described above and a stereo display in which the background was correlated and a pattern of $42 \mathrm{~min}$ arc disparate checks periodically appeared and disappeared. On both VEP and FPL measures, the onset of a response

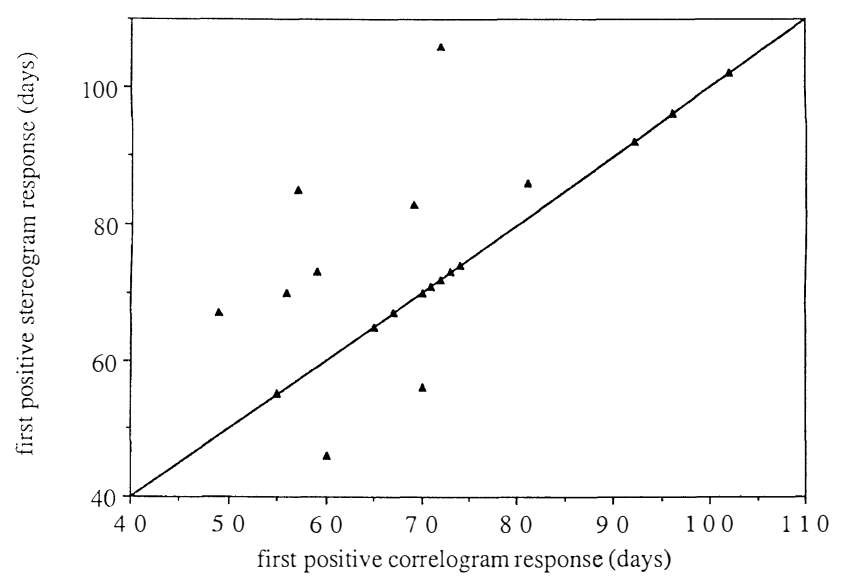

Fig. 3. Comparison in individual infants $(\mathrm{n}=22)$ of the age of onset of $V E P$ responses to random-dot correlograms and random-dot stereograms. Each infant was tested repeatedly at 2-week intervals. The points mark the age at which a VEP was first recorded which gave statistically reliable evidence of a binocular response. The mean age for first response was not significantly different between the two tests (correlogram, 70 days; stereogram, 75 days). (From Smith. ${ }^{7}$ )

to stereo disparity averaged within a few days of the onset of the correlogram response in the same individuals (Fig. 3).

Other groups ${ }^{9-11}$ have concentrated on stereopsis. Birch et al.'s measurements ${ }^{9}$ use an FPL method with line stereograms rather than random dots; they find that infants will show a preference for a display of three vertical bars in which the central bar has a crossed disparity relative to the others, compared with a set of bars which line in a single stereo plane. Despite the difference in stimuli, the onset age for disparity sensitivity (typically $12-16$ weeks) is close to that we find with random-dot displays.

Birch et al. have used their method to test the minimum disparity for which infants show a preference. They find that, after the initial onset of sensitivity, this measure of stereoacuity shows a very rapid increase, typically going from $80 \mathrm{~min}$ arc to $1 \mathrm{~min}$ arc in a matter of 4-5 weeks.

Less familiar than stereoacuity, but equally important in understanding binocular function, is the maximum disparity for which binocular correlation can be detected. Recent work in our group ${ }^{12}$ has tested this through infants' preference for a pattern containing bands of high-disparity random dots on a background of uncorrelated dots. This $D_{\max }$ measure approximately doubles between 16 and 22 weeks of age. Thus the range of infants' disparity sensitivity expands at both its upper and lower limits, although the increase Wattam-Bell ${ }^{12}$ finds for $D_{\max }$ is much more gradual than that reported for $D_{\text {min }}$ or stereoacuity.

\section{DEPTH-RELATED RESPONSES}

Behavioural or VEP demonstrations that infants can discriminate disparity do not, by themselves, prove 
that these disparities convey a sense of depth. It is possible to test this issue, however, when infants start to make reliable reaching responses. It has been shown that 5-month-old infants will use binocular information in determining whether an object is within reach, and in selecting the nearer of two objects as target for a reach. ${ }^{13}$ We have studied the detailed kinematics of 7- to 8-month-olds' reaching for objects, and find a significant improvement in terms of fewer corrective segments of the motion path in binocular compared with monocular viewing. ${ }^{14}$ These results imply that binocular information contributes to the infant's representation of threedimensional space that is used to guide rapid and appropriate actions.

\section{EYE ALIGNMENT}

To detect binocular correlation or disparity, there are two requirements. The eyes must be aligned so that corresponding image points fall on consistent pairings of points in the two eyes, and central binocular neural mechanisms must exist so that information from these paired points can be integrated. Does the onset of functional binocularity reflect the maturation of central mechanisms, or might such mechanisms be present but ineffective because infants' eyes are initially poorly aligned? While eye alignment and vergence control certainly improve with age, there are several lines of evidence that this is not the limiting factor on early binocular function.

First, although there have been reports that large exotropias are common in newborns, ${ }^{15}$ these observations have been criticised as inadequately controlled for versional eye movements and for the large angle kappa of newborn infants. ${ }^{16,17}$ In any case, all studies concur that a substantial proportion of newborns are orthotropic and that this proportion increases with age. Furthermore, appropriate changes of vergence for target distance are observed in the majority of infants before 12 weeks of age. ${ }^{16-18}$ Thus many if not most infants meet the alignment requirements for binocularity well before the age at which binocular performance can be demonstrated.

Second, Birch et al. ${ }^{19}$, which used a periodic version of their three-bar stimulus, could generate the same pattern of disparity differences for stereopsis over a wide range of vergence behaviour by the infant. FPL testing with this display gave a similar distribution of onset age for stereopsis as with the usual version, implying that accurate vergence was not the limiting factor.

\section{PLASTICITY OF BINOCULAR INTERACTIONS}

There is ample evidence, from animal physiology and clinical experience, that the binocular organisation of visual cortex remains highly modifiable for a period after it is initially established. (Daw ${ }^{20}$ provides an upto-date review.) The maintenance of binocular function depends on a critical synergy between the sensory mechanisms that detect binocular relationships and the motor mechanisms that control alignment. Accurate vergence control depends on disparity-sensitive mechanisms to provide the main controlling input; but disparity-sensitive mechanisms can be degraded or destroyed if they do not receive the correlated inputs provided by well-aligned eyes. The commonness of strabismus $^{21}$ in all paediatric neurological conditions shows that this loop is readily broken.

A more subtly disrupting condition is the stress on the accommodation-convergence relationship caused by marked hyperopic refractive error. The clinical concept of accommodative esotropia has arisen from the refractive errors found in children at the time they present with strabismus. However, photo- and videorefractive methods make it possible to screen populations of infants and detect hyperopia before any associated strabismus is manifest. In the follow-up of a large-scale screening programme ${ }^{22-24}$ the approximately $5 \%$ of children who showed significant hyperopia at age 9 months were 13 times more likely to develop strabismus, with an associated breakdown of binocularity, by age 4 years than were controls without significant refractive error. There was also an increased risk of poor acuity among those who had been infant hyperopes. However, the detailed dynamics by which hyperopia leads to a disruption of the sensory-motor binocular loop is still only poorly understood. In a second, current programme, accommodative performance without cycloplegia forms the screening measure. ${ }^{25}$ The outcome of children picked up in this programme, when available, may help us to identify the interactions of accommodation with refraction that create the risk of strabismus.

Is the visual experience associated with wellaligned eyes necessary for the initial establishment of cortical binocularity? Apparently not, according to the results of Birch and Stager. ${ }^{2,26}$ They carried out longitudinal FPL stereo tests with infants with early esotropia, who wore prisms to compensate for the angle of deviation, and found that up to 4 months of age the proportion of esotropic infants showing disparity sensitivity was similar to that of a normal group. However, beyond this age the number giving positive results increased in the normal group but steeply declined among the esotropes. Thus aligned binocular input was necessary to sustain binocular mechanisms in this group even though it was not necessary for their initial development.

Birch and Stager's result also implies that an absence of sensory binocularity was not generally the cause of early esotropic deviation. This is consistent with the results of Wattam-Bell et al. ${ }^{4}$ who followed a 


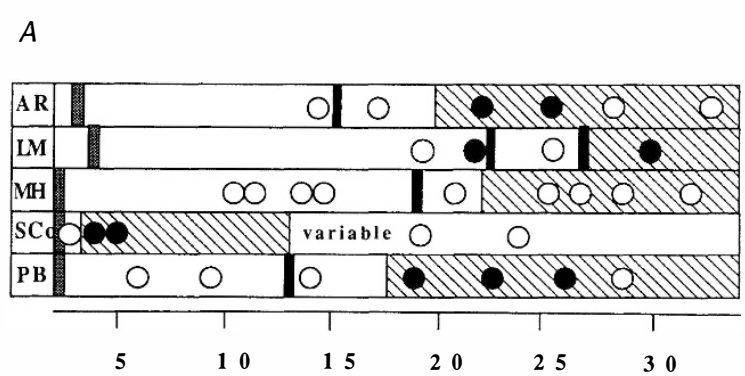

$B$
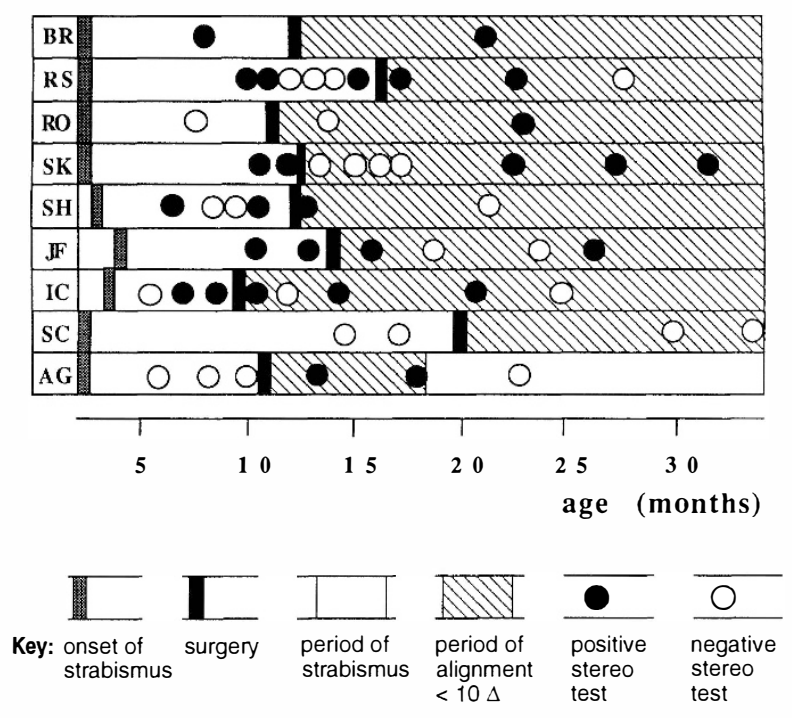

Fig. 4. Results of FPL stereogram testing on individual strabismic children. (A) Esotropic children who consistently used a particular eye for fixation. (B) Children who could fixate with either eye (all esotropic except for exotropic BR). Filled and open circles show ages at which positive and negative results, respectively, were obtained, relative to the times of surgery on the presence of a deviation (see key). (From Smith et al. ${ }^{29}$ )

group of infants whose family history put them at risk for strabismus. Among this group were infants who showed positive responses on the correlogram VEP test between 11 and 20 weeks but developed manifest strabismus before 6 months. The results of both studies suggest that the sensory-motor loop was initially disrupted on the motor rather than the sensory side. This is not to deny, of course, the existence of conditions where a failure of sensory binocularity is primary - for example where the visual pathways are congenitally misrouted. ${ }^{27,28}$

Does the plasticity of the binocular system necessarily mean that misalignment destroys sensory binocularity? An alternative possibility is that binocular interactions may be set up between points in the two eyes' fields that correspond at the angle of deviation, and there is evidence that this occurs. A group of children, with onset of strabismus in the first year and surgery before age 2 years, has been studied longitudinally with the FPL random-dot stereogram test in our unit. ${ }^{29}$ Children who showed a strongly preferred eye for fixation generally gave negative

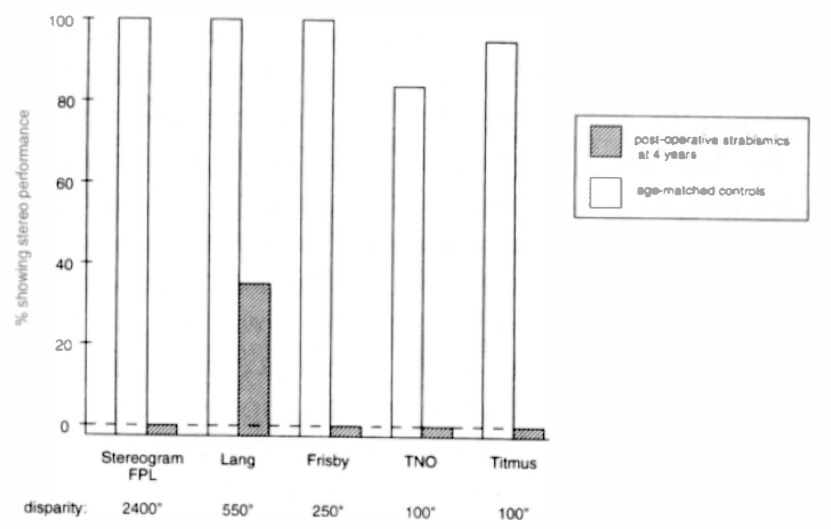

Fig. 5. Results of a variety of stereo tests at age 4 years on a group of the surgically corrected strabismic children $(\mathrm{n}=$ 14) whose earlier results are shown in Fig. 4, compared with age-matched orthotropic controls $(\mathrm{n}=18)$.

results pre-operatively. However, among the children who could fixate with either eye, the majority showed responses to stereo disparity (Fig. 4). There was no prism correction for the deviation during these tests, so these results imply binocular interactions corresponding to the angle of deviation, which was typically in the range 30-60 prism dioptres.

Following surgical correction (and orthoptic therapy), the majority of children in each group gave positive results on the FPL stereo test. This implies that at the age of surgery (11-27 months) the system is sufficiently plastic to re-establish binocular interactions beween the newly aligned corresponding points. However, this appears to be a transient effect. We have re-examined many of these children at age 4 years, with a variety of stereo tests ${ }^{40}$ (Fig. 5). There is essentially no evidence of persisting stereopsis, even on the FPL test for which the same children had shown positive responses about 2 years earlier. A similar conclusion was reached by Charles and Moore $^{30}$ from clinic follow-up of an extended version of the same group. The reasons for the failure to maintain binocularity are unclear: possibly the reestablished or modified binocularity is weak or fragmentary, and so is vulnerable to disruptions of sensory-motor synergy which a better-established system could withstand; possibly the forces leading to the original strabismus persist, although the surgical and orthoptic therapy may mean that their oculomotor consequences are less visible.

\section{UNANSWERED QUESTIONS}

The progress that has been made in understanding how binocularity develops makes it possible to formulate a number of unanswered questions.

How is the Infant's Visual System Organised Before
the Establishment of Binocularity?

Anatomically, fibres carrying signals from both eyes have established terminations in the striate cortex 
well before birth, ${ }^{31}$ but their separation into ocular dominance columns is not complete for some weeks postnatally. ${ }^{32,33}$ If the fibres make cortical connections but do not provide the basis for binocular interaction, how are their binocular relationships organised before the onset of stereopsis? A simple hypothesis might be that individual cortical cells do not combine signals from the two eyes. There appears to be little direct evidence on this point from primate developmental physiology. However, in the young kitten ${ }^{34}$ the evidence is against this idea: before the segregation of ocular dominance columns, many layer IV cells are binocular, unlike in the adult. Held $^{35}$ proposes that such convergence characterises an initial state of 'primitive binocularity' in which the two eyes' inputs are simply summed at the earliest cortical stage. On this view, development of disparity sensitivity depends on separating left- and right-eye signals in layer IV, so that they can come to interact in a range of disparity-specific neurons in the upper cortical layers.

However, it is difficult to see how 'primitive binocularity' can be reconciled with the development of sensitivity to random-dot correlograms. A system of neurons which summed signals from the two eyes would be very strongly stimulated by the transitions from anticorrelated to correlated dots, which would resemble transitions between high- and low-contrast dots. Indeed, this is exactly the appearance if presentation of the dot patterns is not separated between the eyes, a condition which elicits strong VEPs in pre-stereoptic infants. ${ }^{3,6}$ Thus it might be expected that correlogram responses, behavioural and VEP, would be observed in the stage of primitive binocularity, with disparity-sensitive responses appearing later. In direct comparison (Fig. 3) this dissociation was not found. Perhaps binocular inputs are combined in the pre-stereoptic infant but in a way which is not spatially specific as would be required for point-by-point summation. At present, the existence and nature of pre-stereoptic binocularity remain uncertain.

\section{How Does the Young Infant Control Vergence?}

We have reviewed evidence that infants before 3 months can maintain eye alignment and adjust it to converge on a near target. In the mature system, a disparity signal provides the main input to achieve this, but these infants are insensitive to disparity. Held $^{35}$ suggests that maximisation of the signal in primitive binocular summing neurons might guide vergence; but this is exactly the signal which, as discussed above, we might expect to see in the correlogram VEP and do not. Alternatively, if separate monocular signals are available in the visual system of the pre-stereoptic infant, foveal fixation of a target might be controlled for each eye separately, leading secondarily to binocular alignment. Given the importance of the superior colliculus in guiding fixation, this might be somewhat distinct from the issue of whether monocular signals are separate in the visual cortex. Finally, it has been suggested that accommodative vergence ${ }^{36}$ may contribute to infants' vergence control. Aslin and Jackson ${ }^{37}$ showed that accommodative demand could drive vergence changes in infants as young as 2 months. However, it seems unlikely that signals related to accommodation could provide the accuracy needed to maintain eye alignment; and if it were supposed to be the main source of early vergence control, it raises a puzzling question of how accommodation-vergence relationships came to be calibrated in the first case.

\section{What Underlies the Increase in Stereoacuity and $D_{\text {max }}$ ?}

Of these, $D_{\max }$ is the easier to understand, at least speculatively. Disparity detection requires the interaction of signals from neighbouring points in the combined topographic representation of the binocular field of view. It is reasonable to imagine progressive growth of the lateral intracortical connections serving this interaction. The parallel developmental increases in $D_{\max }$ for stereopsis and for motion detection ${ }^{12}$ suggest that a general pattern of extending connections might be responsible for both.

However, stereoacuity is reported to increase at a much more rapid pace than $D_{\text {max }}$. This increase is also many times faster than the increase in resolution acuity with age, and faster than the increase in vernier acuity (which itself outpaces the increase in resolution acuity ${ }^{38}$ ). Thus the development of stereoacuity does not reflect the broader improvement in precision of spatial information available in the visual system. Either a range of fine-tuned disparity detectors is arising in cortex, by a developmental process specific to disparity, at a remarkable pace; or the necessary range of detectors is established together at the onset of stereoposis, and the infant takes a comparatively short time to organise and interpret their patterns of output.

Binocularity has been a central topic in the study of visual development for two reasons. First, the delicate sensory-motor synergy underlying binocular function means that breakdown of this relationship provides the commonest developmental disorders of vision, and so the area is central as a scientific underpinning for paediatric ophthalmology. Second, binocularity involves the clear interaction of independent streams of information from the two eyes; this has made it a model system for considering much broader issues of brain development and organisa- 
tion, in particular how the brain adapts to patterns of correlation across multiple inputs. ${ }^{39}$ For these reasons, the unanswered questions of binocular development will no doubt continue to be a focus of research.

Research described here is the result of work by many in the Visual Development Unit, in particular Janette Atkinson, John Wattam-Bell, Jocelyn Smith and Shirley Anker. We thank Peter Watson and Tony Moore for clinical collaboration. This work is supported by a programme grant from the Medical Research Council.

Key words: Infant vision, Visual development. Binocular vision, Strabismus, Stereopsis.

\section{REFERENCES}

1. Braddick OJ, Atkinson J. Some recent findings on the development of human binocularity: a review. Behav Brain Res 1983;10:141-50.

2. Birch EE. Stereopsis in infants and its developmental relation to visual acuity. In: Simons K, editor. Early visual development: normal and abnormal. New York: Oxford University Press, 1993.

3. Braddick OJ, Wattam-Bell J, Day J, Atkinson J. The onset of binocular function in human infants. Hum Neurobiol 1983;2:65-9.

4. Wattam-Bell J, Braddick OJ, Atkinson J, Day J. Measures of infant binocularity in a group at risk for strabismus. Clin Vision Sci 1987;1:327-36.

5. Julesz B, Kropfl W, Petrig B. Large evoked potentials of dynamic random-dot correlograms and stereograms permit quick determination of stereopsis. Proc Natl Acad Sci USA 1980;77:2348-51.

6. Braddick OJ, Atkinson J, Julesz B, Kropfl W, BodisWollner I, Raab E. Cortical binocularity in infants. Nature 1980;228:363-5.

7. Smith J. The development of binocular vision in normal and strabismic infants. PhD thesis, University of Cambridge, 1989.

8. Smith J, Atkinson J, Braddick OJ, Wattam-Bell J. Development of sensitivity to binocular correlation and disparity in infancy. Perception 1988;17:365.

9. Birch EE, Gwiazda J, Held R. Stereoacuity development for crossed and uncrossed disparities in human infants. Vision Res 1982;22:507-13.

10. Fox R, Aslin RN, Shea SL, Dumais ST. Stereopsis in human infants. Science 1980;207:323-4.

11. Held R, Birch EE, Gwiazda J. Stereoacuity of human infants. Proc Natl Acad Sci USA 1980;77:5572-4.

12. Wattam-Bell J. Stereoscopic and motion $D_{\max }$ in adults and infants. Invest Ophthalmol Vis Sci 1995;36:S910.

13. Yonas A, Granrud C. Reaching as a measure of infants' spatial perception. In: Gottlieb G, Krasnegor $\mathrm{N}$, editors. Measurement of audition and vision in the first year of life. Norwood, NJ: Ablex, 1985:301-22.

14. Atkinson J, Braddick OJ, Hood B. Kinematics of infants reaching with monocular and binocular vision. Infant Behav Dev 1994;17:504.

15. Archer SM. Detection and treatment of congenital esotropia. In: Simons K, editor. Early visual development: normal and abnormal. New York: Oxford University Press, 1993:

16. Hainline L, Riddell PM. Eye alignment and convergence in young infants. In: Vital-Durand F, Atkinson J,
Braddick O, editors. Infant vision. Oxford University Press (in press).

17. Hainline L, Riddell PM. Binocular alignment and vergence in early infancy. Vision Res 1995;35: 3229-36.

18. Aslin RN. Infant accommodation and convergence. In: Simons K, editor. Early visual development: normal and abnormal. New York: Oxford University Press, 1993.

19. Birch EE, Gwiazda J, Held R. The development of vergence does not account for the development of stereopsis. Perception 1983;12:331-6.

20. Daw N. Visual development. New York: Plenum Press, 1995.

21. Atkinson J, Van Hof-van-Duin J. Assessment of normal and abnormal vision during the first years of life. In: Fielder A, Bax M, editors. Management of visual handicap in childhood. London: MacKeith Press.

22. Atkinson J. Infant vision screening: prediction and prevention of strabismus and amblyopia from refractive screening in the Cambridge photorefraction programme. In: Simons K, editor. Early visual development: normal and abnormal. New York: Oxford University Press, 1993.

23. Atkinson J, Braddick OJ, Durden K, Watson PG, Atkinson S. Screening for refractive errors in 6-9 months old infants by photorefraction. Br J Ophthalmol 1984;68:105-12.

24. Atkinson J, Braddick O, Bobier W, Anker S, Ehrlich D, King J, Watson PG, Moore AT. Two infant vision screening programmes: prediction and prevention of strabismus and amblyopia from photo- and videorefractive screening. Eye 1996;10:189-98.

25. Anker SE, Atkinson J, Braddick OJ, Ehrlich DE, Weeks F, Wade J. Accommodative measures of ametropia from video-refractive screening of a total infant population. Invest Ophthalmol Vis Sci (Suppl) 1995;36:S48.

26. Birch EE, Stager DR. Monocular acuity and stereopsis in infantile esotropia. Invest Ophthalmol Vis Sci 1985;26:1624-30.

27. Apkarian P. Chiasmal crossing defects in disorders of binocular vision. Eye 1996;10:222-32.

28. Guillery RW. The reasons for the loss of binocularity in albinism. Eye 1996;10:217-21.

29. Smith JC, Atkinson J, Anker S, Moore AT. A prospective study of binocularity and amblyopia in strabismic infants before and after corrective surgery: implications for the human critical period. Clin Vision Sci 1991;6:335-53.

30. Charles SJ, Moore AT. Results of early surgery for infantile esotropia in normal and neurologically impaired infants. Eye 1992;6:603-6.

31. Rakic P. Prenatal development of the visual system in rhesus monkey. Phil Trans R Soc Lond B 1977; 278:245-60.

32. Hubel DH, Wiesel TN. Functional architecture of macaque monkey striate cortex. Proc R Soc Lond B 1977;198:1-59.

33. Horton JC, Hedley-White T. Mapping of cytochrome oxidase patches and ocular dominance columns in human visual cortex. Phil Trans $\mathrm{R}$ Soc Lond B 1984;304:252-72.

34. LeVay S, Stryker MP, Sherk H. Ocular dominance columns and their development in layer IV of the cat's visual cortex: a quantitative study. J Comp Neurol 1978;179:223-44.

35. Held R. Two stages in the development of binocular vision and eye alignment. In: Simons K, editor. Early 
visual development: normal and abnormal. New York: Oxford University Press, 1993.

36. Judge SJ. How is binocularity maintained during convergence and divergence. Eye 1996;10:172-6.

37. Aslin RN, Jackson RW. Accommodative convergence in young infants: development of a synergistic sensorymotor system. Can J Psychol 1979;33:222-31.

38. Shimojo S, Birch EE, Gwiazda J, Held R. Development of vernier acuity in infants. Vision Res 1984; 24:721-4.
39. Braddick OJ. Binocular vision as a model developmental system. Ophthalmic Physiol Optics 1989;9:466.

40. Atkinson J. The Cambridge assessment and screening of vision in 'high risk' infants and young children. In: Anastasiow NJ, Harel S, editors. At-risk infants: interventions, families, and research. Baltimore: Paul H Brookes, 1993.

41. Wattam-Bell J. Analysis of infant visual evoked potentials (VEPs) by a phase-sensitive statistic. Perception 1985;14:A33. 